\title{
TIME-OPTIMIZED SOFTWARE IMPLEMENTATION OF THE PRESENT CIPHER ON PYTHON LANGUAGE
}

\section{FERNANDO MARTÍNEZ S., HOLMAN MONTIEL A. \& EDWAR JACINTO G}

Technology Faculty, Universidad Distrital Francisco José de Caldas, Bogotá, Colombia

\begin{abstract}
This paper shows the development of the PRESENT cipher using Python 3 language, this cipher uses 64-bit data and 80-bits or 128-bit keys. The main objetive is optimizing the execution time of the algorithm in order to be used along with different kinds of embedded systems such as microcontrollers, SoCs (System on Chip) or even programable logic devices like FPGA (Field Programable Gate Array) for IoT (Internet of Things) and/or smart sensor networks applications. First, some bit-wise operations which are commonly used in cryptographic algorithms were implemented and tested for defining which must be used in the final implementation. After, three different approaches of implementing the complete PRESENT cipher on Python were proposed, the third version reached a time reduction from $25.3 \%$ to $45.3 \%$ compared with the other two versions. These time reduction percents depend on the kind of operation (cipher or decipher) and the width of the key (80-bit or 128-bit). Finally, the algorithm implementation obtained shown that it is able to be used on the applications previously mentioned as well as it can be used on embeded systems which can run a Python interpreter.

KEYWORDS: Embedded Cryptography, Lightweight Block Cipher, Python, PRESENT Cipher
\end{abstract}

Received: Oct 06, 2020; Accepted: Oct 26, 2020; Published: Dec 03, 2020; Paper Id.: IJMPERDOCT202083

\section{INTRODUCTION}

Nowadays, the interconnection between electric, electronic and informatics system is kind of a general rule, due to the automation degree of the systems presented in most of the industrial and home technology applications, such as Smart Grid, IoT (Internet of Things) and IIoT (Gunduz \& Das, 2020) ,(Ahad et al., 2020),(Alcaraz, 2019). All of those information systems have to ensure its integrity and safeness, that is why cryptography keeps being one of the most researched areas and new algorithms and methods are continuously developed (Zhao et al., 2020),(Adomnicai et al., 2019),(Ali, 2017),(Lang Li et al., 2018),(Gnatyuk et al., 2019), in order to be used in different data management applications (Sutanto, 2019),(ÖzÇakmak et al., 2019),(Atiewi et al., 2020). Cryptographic algorithms are used to being computationally "heavy", expending a lot time ciphering and deciphering the information. This fact is not event a huge issue on PC-based applications, but in most of IoT or embedded applications that problem could be critical due to the restrictions of the used processors. For that reason embedded information systems usually implements their security modules using lightweight or ultra-lightweight ciphers (Bansod et al., 2016),(Ghafari et al., 2016),(L Li et al., 2016), which are cryptographic algorithms designed to be used on processors or digital systems of low or middle computing performance. One of those ultra-lightweight ciphers is PRESENT(Bogdanov et al., n.d.), which has been implemented on several embedded applications (Muduvangattil, 2018)(Jithendra \& Shahana, 2020)(Azari \& Dr. Prashant V Joshi, 2018),(Eisenbarth et al., 2017). PRESENT is a block cipher that has an 80-bit and a 128-bit key versions, both of them has been widely implemented, it processes 64-bit data blocks. In SIE and ARMOS research 
groups from Universidad Distrital (Bogotá, Colombia), some papers about Cryptography has been published, especially in relation to lightweight and ultra-lightweight cipher implementation over several embedded platforms like FPGA (Field Programmable Gate Array), microcontrollers and SoC (System on Chip). Among the ciphers implemented are CLEFIA and PRESENT.

Generally, the IoT or embedded applications that implements ultra-lightweight cipher as information security modules, communicate with a central computer which has to be able to cipher and decipher information using the same cryptographic algorithm that implies that software implementations always exist, but usually these are not as optimized as the hardware or firmware implementations. There are several software cryptographic libraries to use on several languages such as $\mathrm{C} / \mathrm{C}++$, Java and Python (Eijs, 2020), but in general they includes the standard ciphers as AES but not lightweight ciphers, these ones are usually implemented separately (Setetemela et al., 2019), (Rahouma et al., 2020). The aim of this paper is to develop a time-optimized python implementation for the PRESENT algorithm to be used along with embedded systems on embedded information security systems.

\section{METHODOLOGY}

PRESENT is a block cipher that processes 64-bit data blocks using 80-bit or 128-bit keys, that ciphering process takes 32 processing rounds, this procedure is summarised in the block diagram shown in the Fig. 1, it executes 31 identical rounds and a final simple round.

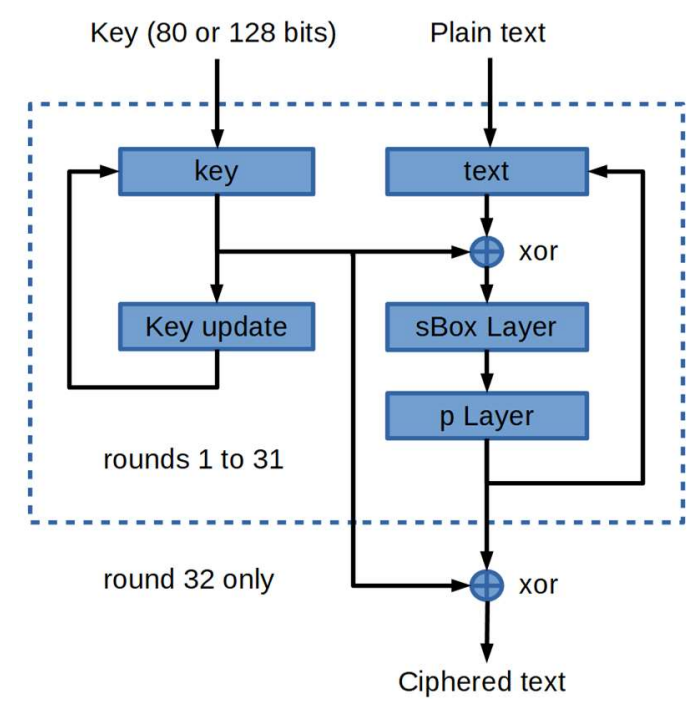

Figure 1: General block diagram of the PRESENT cipher working.

The block diagram shows the following main blocks: XOR operators, sBox Layer, $p$ Layer and Key update; the first ones perform and XOR operation between the data block and the 64 least significant bits of the key block, the second one changes the information in 4bit groups or nibbles defined as $x$, and produces the output $S[x]$ as shown in Table 1 . The third main block is the permutation layer $p$ Layer, it changes the position of each bit on the data block as shown in Table 2, where $i$ is the original position and $P(i)$ is the resultant one. Finally, the Key update block works different depending on the type of key used: 80-bit or 128-bit. The updating of an 80-bit key consists of the following steps:

- the current key is left-rotated 61 bits,

- the 4 most significant bits of the resultant key is passed through the sBox Layer, 
- the group of 5 bits in the positions from 19 to 15 of the obtained key, is applied a XOR function with the round counter.

Table 1: sBox Layer Nibble Replacement Values[Hex]

\begin{tabular}{|l|l|l|l|l|l|l|l|l|l|l|l|l|l|l|l|l|}
\hline $\boldsymbol{x}$ & 0 & 1 & 2 & 3 & 4 & 5 & 6 & 7 & 8 & 9 & $\mathrm{~A}$ & $\mathrm{~B}$ & $\mathrm{C}$ & $\mathrm{D}$ & $\mathrm{E}$ & $\mathrm{F}$ \\
\hline $\boldsymbol{S}[\boldsymbol{x}]$ & $\mathrm{C}$ & 5 & 6 & $\mathrm{~B}$ & 9 & 0 & $\mathrm{~A}$ & $\mathrm{D}$ & 3 & $\mathrm{E}$ & $\mathrm{F}$ & 8 & 4 & 7 & 1 & 2 \\
\hline
\end{tabular}

On the other had, the updating of 128 -bit keys is very similar; it takes the following steps:

- the current key is left-rotated 61 bits,

- the 8 most significant bits of the resultant key is passed through the sBox Layer in two groups of 4 bits,

- the group of 5 bits in the positions from 66 to 62 of the obtained key, is applied a XOR function with the round counter.

All of this design is related to the hardware implementation, and it was the inspiration for the first software implementation done in $\mathrm{C}$ language.

Table 2: pLayer Permutation Bit Position

\begin{tabular}{|l|l|l|l|l|l|l|l|}
\hline $\boldsymbol{i}$ & $\boldsymbol{P}(\boldsymbol{i})$ & $\boldsymbol{i}$ & $\boldsymbol{P}(\boldsymbol{i})$ & $\boldsymbol{i}$ & $\boldsymbol{P}(\boldsymbol{i})$ & $\boldsymbol{i}$ & $\boldsymbol{P}(\boldsymbol{i})$ \\
\hline 0 & 0 & 16 & 4 & 32 & 8 & 48 & 12 \\
\hline 1 & 16 & 17 & 20 & 33 & 24 & 49 & 28 \\
\hline 2 & 32 & 18 & 36 & 34 & 40 & 50 & 44 \\
\hline 3 & 48 & 19 & 52 & 35 & 56 & 51 & 60 \\
\hline 4 & 1 & 20 & 5 & 36 & 9 & 52 & 13 \\
\hline 5 & 17 & 21 & 21 & 37 & 25 & 53 & 29 \\
\hline 6 & 33 & 22 & 37 & 38 & 41 & 54 & 45 \\
\hline 7 & 49 & 23 & 53 & 39 & 57 & 55 & 61 \\
\hline 8 & 2 & 24 & 6 & 40 & 10 & 56 & 14 \\
\hline 9 & 18 & 25 & 22 & 41 & 26 & 57 & 30 \\
\hline 10 & 34 & 26 & 38 & 42 & 42 & 58 & 46 \\
\hline 11 & 50 & 27 & 54 & 43 & 58 & 59 & 62 \\
\hline 12 & 3 & 28 & 7 & 44 & 11 & 60 & 15 \\
\hline 13 & 19 & 29 & 23 & 45 & 27 & 61 & 31 \\
\hline 14 & 35 & 30 & 39 & 46 & 43 & 62 & 47 \\
\hline 15 & 51 & 31 & 55 & 47 & 59 & 63 & 63 \\
\hline
\end{tabular}

\subsection{Python Implementation}

The software implementation of the PRESENT cipher on Python language (3.7 version) was done testing some different approaches, but in general all of them follow the flow chart shown in Fig. 2. As the Key updating does not depend on the data block, is it possible to compute the key for each round independently, this fact helps reducing the execution time of the ciphering and deciphering processes. Another important feature is that the software implementation makes difference between the first round and the rest (from 2 to 32), unlike the block diagram of Fig. 1 where the different round is the last one. 


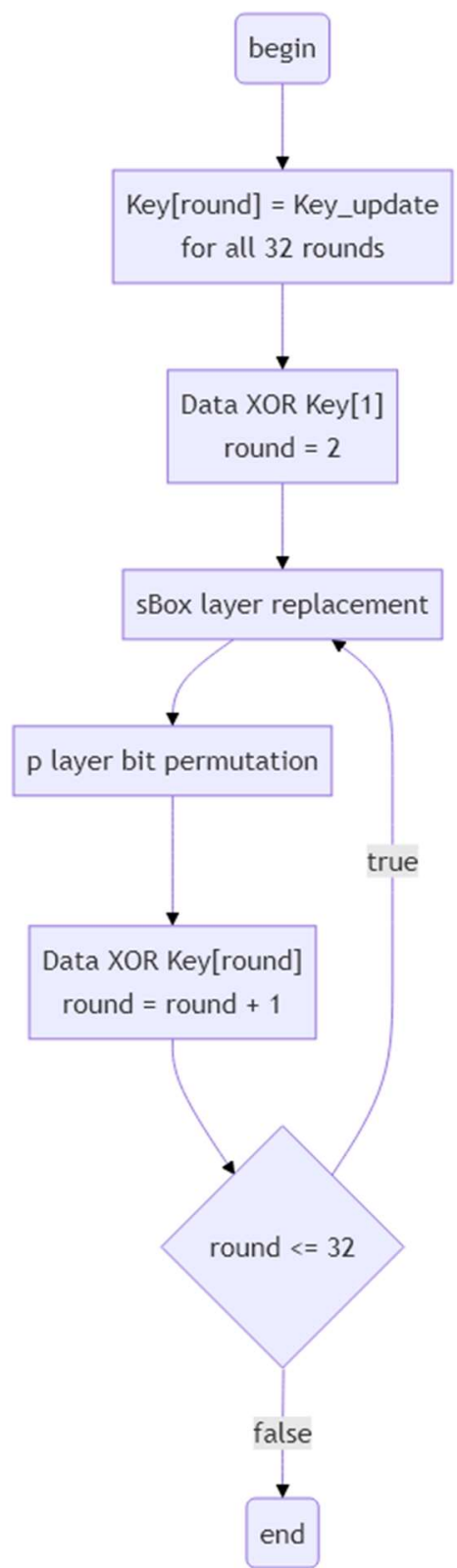

Figure 2: Flow chart of the PRESENT cipher Python implementation.

\section{RESULTS AND DISCUSSIONS}

A first software implementation was done on $\mathrm{C}$ language inspired on the one performed on several embedded systems based on micro-controllers of 8, 16 and 32 bits, and these ones in turn was based on the hardware (FPGA) implementation. That cipher is totally functional but it is not time optimized at all, due to not all the operation performed on a programmable hardware device have the same time execution behaviour that the ones performed on software. In order to integrate the PRESENT developments previously done with the software GUI done in Python, it was necessary to implement the complete cipher on this language. Python is an interpreted scripting language, for that reason the programs written on this are used to run slower than the ones written on a complied language like $\mathrm{C}$, that is why it is imperative to optimise it. Three different Python implementations were done and compared themselves in order to obtain the best time performance. The computer 
used for doing all of the tests done including the different cipher implementations has the next features: CPU: 64-bit 2.8GHz 4-core $\mathrm{x} 86$-based and RAM: 12GB.

\subsection{Time Optimization}

All of the implementations of lightweight ciphering algorithms works at the level of bits, using repetitively shifting and masking operation, that is why a time analysis over the most used bit-wise operations was done. The shift operations where compared against the same functionality performed through the multiply or division on powers of two as shown in equations 1 and 2, where $n$ is the number of position to be shifted, in this case both equations show a right shifting.

$$
\begin{aligned}
& B=A \gg n(1) \\
& B=\left(\frac{A}{2^{n}}\right)-1
\end{aligned}
$$

Using timeit Python library, the execution times of the shifting operations shown in equations 1 and 2 where calculated. Each algorithm was tested over 128-bit variables performing a 64-bit shifting and repeated 10000 times, averaging the resultant values. On the other hand, another frequently used operation is the bit masking, most of times it is desired to mask a small group of bits. This operation was performed in two different way, applying a mask of the complete number of bits or doing a mask only with the necessary bits and shift it to the desired position. The equations 3 and 4 show the correspondent masking algorithms that was tested in the same way to the previous ones.

$$
\begin{aligned}
& B=A \text { and }\left(\operatorname{not} M_{k}\right)_{(3)} \\
& B=A \operatorname{and}\left(\operatorname{not}\left(M_{r} \ll n\right)\right)_{(4)}
\end{aligned}
$$

The and operation and not operation shown in equations 3 and 4 refer to bit-wise operations, $M_{k}$ is the mask of complete number of bits (128 for the tests), $M_{r}$ is reduced mask to the desired number of bits, and finally $n$ is the number of bits to be shifted.

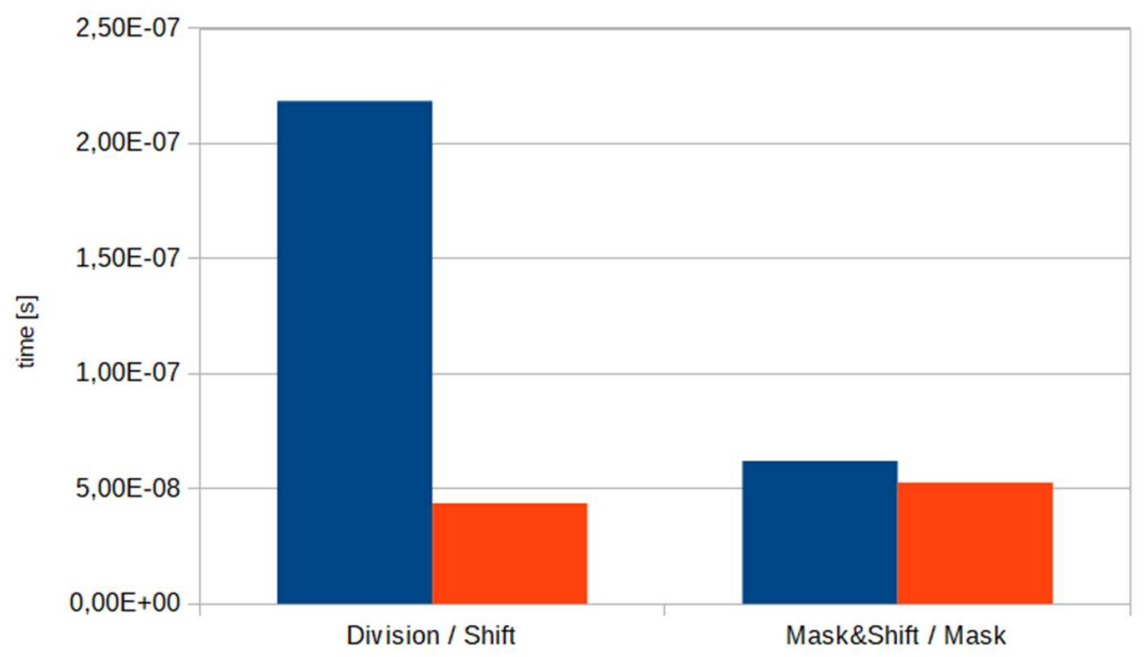

Figure 3: Execution times for division or shifting operations (left) and masking + shifting or only masking operations (right). 
Figure 3 shows the execution time for the division operations against shifting operation and also the operation of masking only the necessary bits and shift them against masking the complete number of bits. As a result, to do shifting operations spent around $80 \%$ less time than to do divisions, on the other hand, masking the complete number of bits spent around $15 \%$ less time than implementing masking/shifting operations, that is why these methods were used in next steps described in this paper.

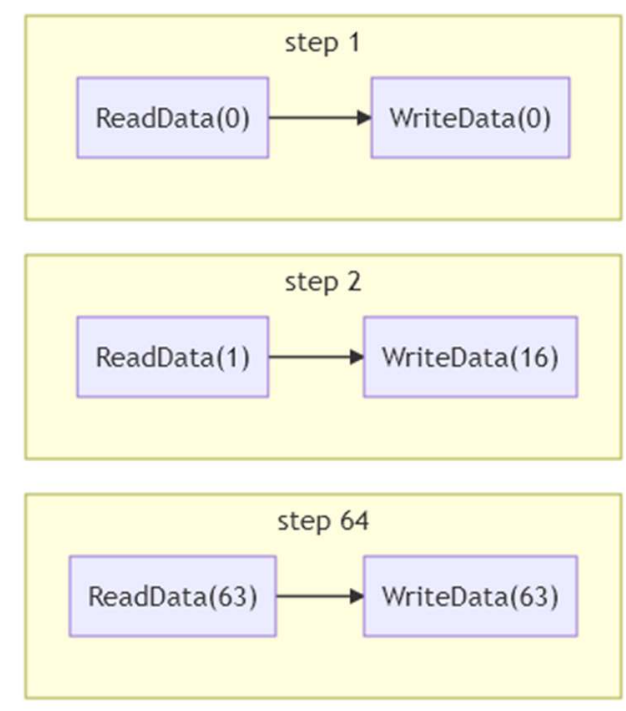

Figure 4: pLayer implementation for the first version of the PRESENT cipher.

\subsection{Implementation Approaches}

Three different implementation approaches were tested in order to select the optimal, that means the one with the lower execution time. Each of them are described as follows.

1) Based on the $C$ version: This implementation is based on the one done in $\mathrm{C}$ language which in turn was based on the hardware VHDL (Very high speed Hardware Description Language) implementation. Especially the $p$ Layer was done by read and write bit functions as shown in Figure 4.

Where the parameter received for the functions is the bit number for being permuted and corresponds to the $p$ Layer. Each function is implemented by means of using bit shifting and masking operations.

2) Modified $p$ Layer version: This implements a table for the $p$ Layer indicating the bit permutation. The operation is performed by means of an initial mask with only an active bit, which is shifted a specific number of bits according to the $p$ Layer table as shown in Figure 5. 


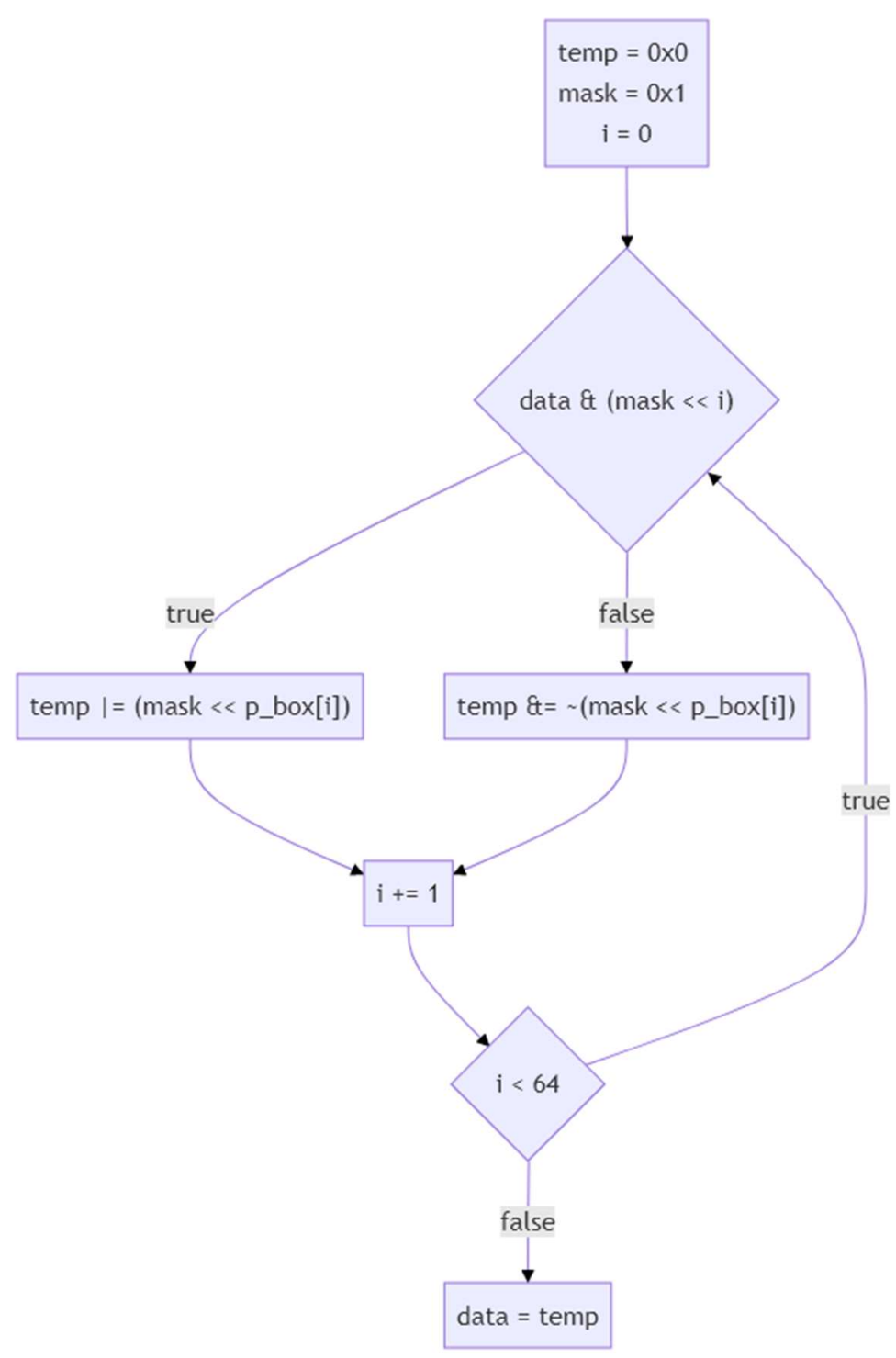

Figure 5: pLayer implementation for the second version of the PRESENT cipher.

3) Round key generation version: This is based on the Modified p Layer version but it calculates the keys for all of the rounds previous to start the encryption or decryption operations as it is shown in the example for an 80-bit cipher shown in Fig. 6. 


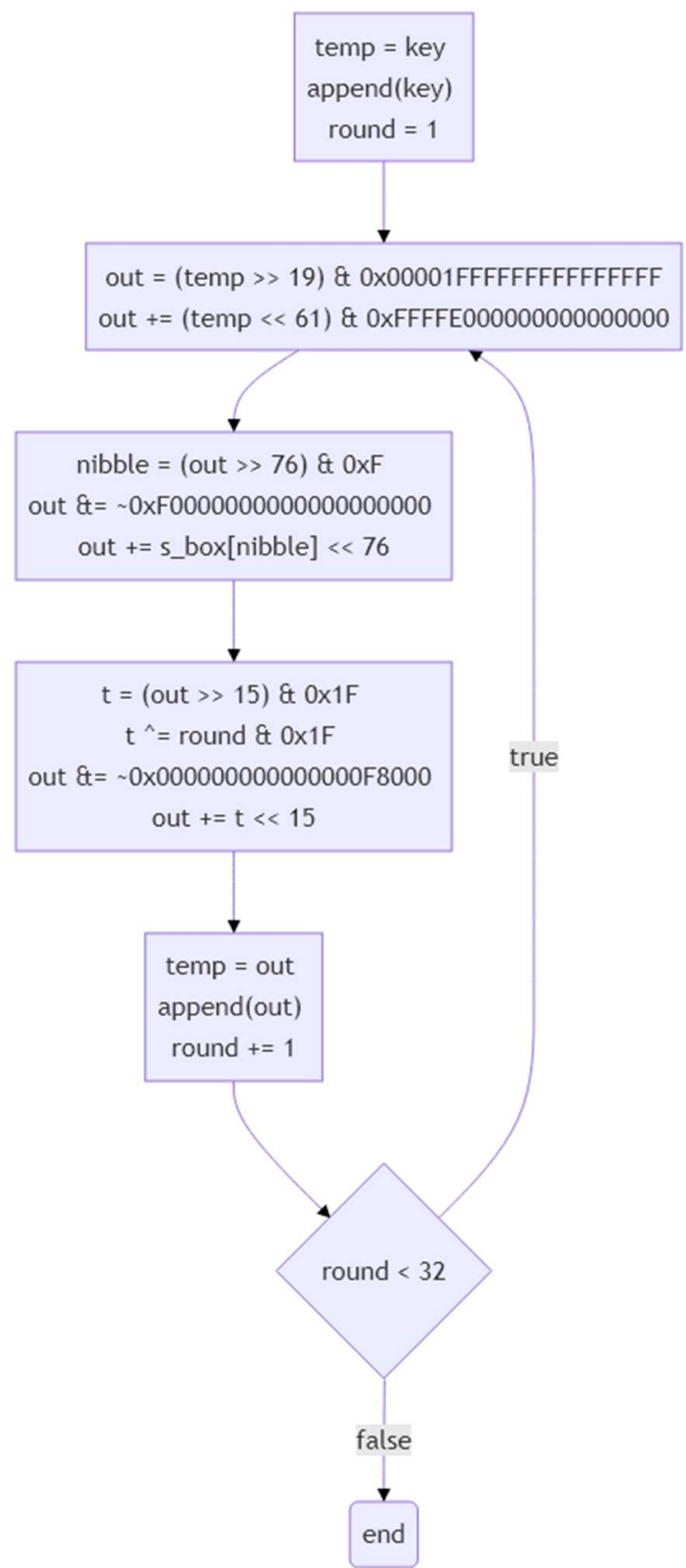

Figure 6: pLayer implementation for the second version of the PRESENT cipher

Over the three different implementations, time executing measurements were done, the Fig. 7 shows the comparison between the 3 implementations approaches for the 80-bit version of the PRESENT algorithm and the Fig. 8 shows the correspondent for the 128-bit version. Each test performed 500 times the same ciphering/deciphering task, averaging all of those obtained times. 


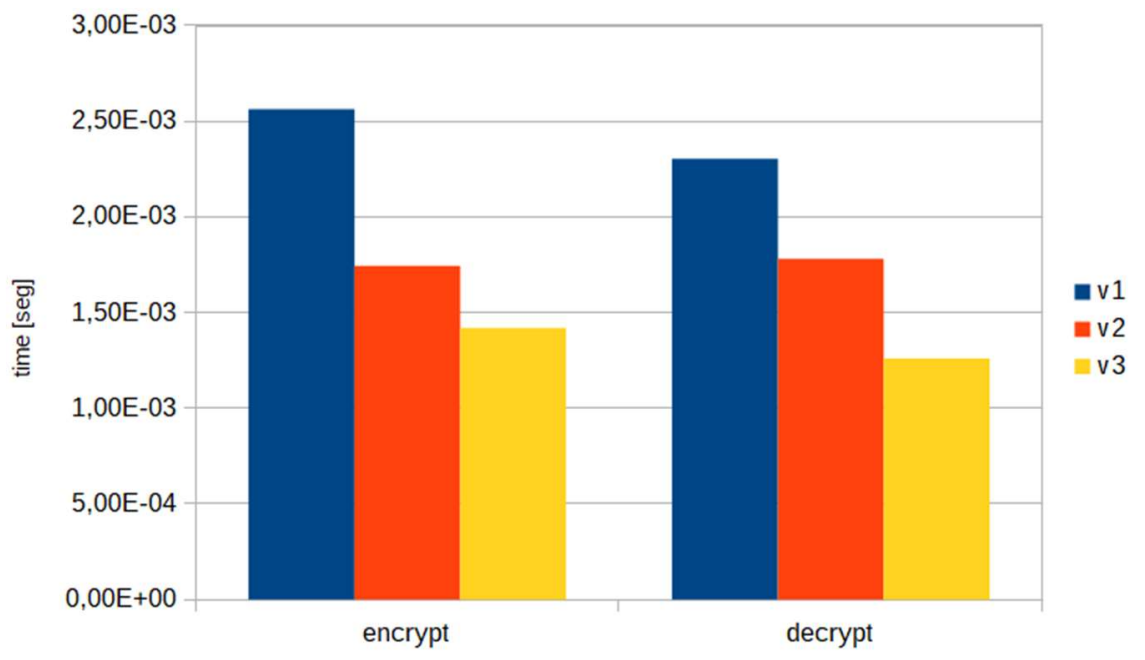

Figure 7: Execution timer of the ciphering (left) and ciphering operation (right) for the 3 versions of the PRESENT 80 cipher.

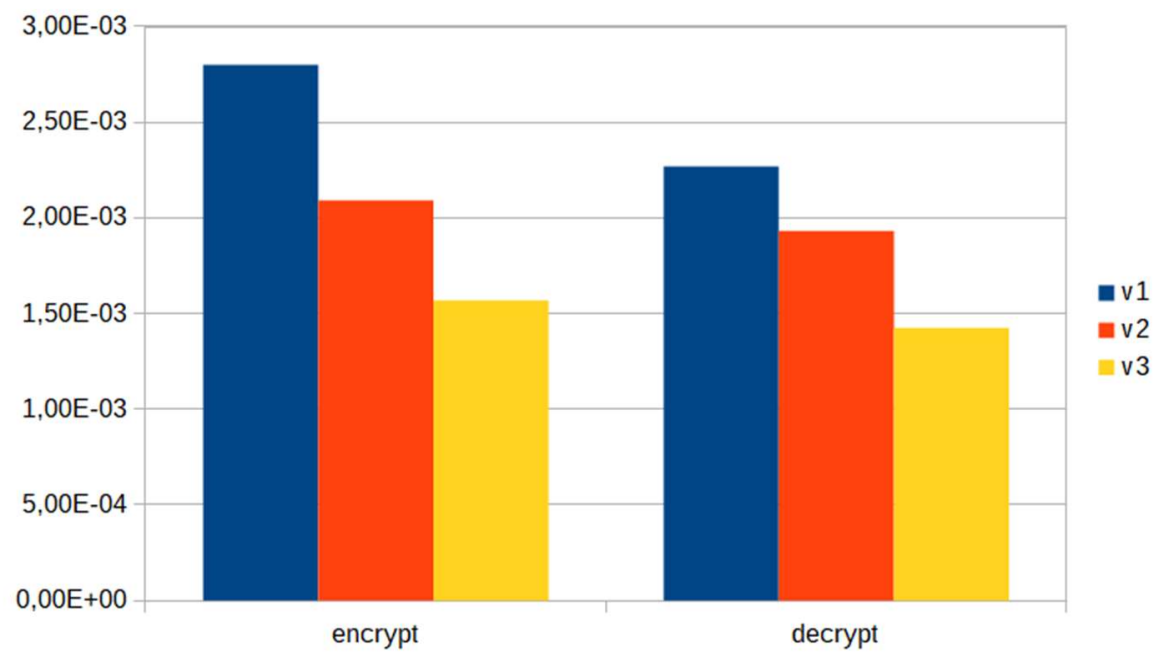

Figure 8: Execution timer of the ciphering (left) and ciphering operation (right) for the 3 version of the PRESENT 128 cipher.

As a result, the comparison done over the three different implementation approaches shows clearly than the previous described version 2 (modified $p$ Layer) and the version 3 (Round key generation) both improve the time performance against the version 1 . The Table 3, shows the time reduction reached by v2 and v3 versions compared with the version 1 , for both ciphering (encrypt) and deciphering (decrypt) operations for the PRESENT cipher that uses 80-bit key, those values are between $22.7 \%$ and $31.9 \%$ for the version 2 and between $44.6 \%$ and $45.3 \%$ for the version 3 .

Table 3: Time Reduction With The Version 2 \& 3 (PRESENT 80)

\begin{tabular}{|l|l|l|}
\hline Operation & $\boldsymbol{v} 2$ & $\boldsymbol{v 3}$ \\
\hline Encrypt & $31,9 \%$ & $44,6 \%$ \\
\hline Decrypt & $22,7 \%$ & $45,3 \%$ \\
\hline
\end{tabular}


Table 4: Time Reduction with the PRESENT 128 cipher

\begin{tabular}{|l|l|l|}
\hline Operation & $\boldsymbol{v} 2$ & $\boldsymbol{v 3}$ \\
\hline Encrypt & $25,3 \%$ & $44 \%$ \\
\hline Decrypt & $14,8 \%$ & $37,1 \%$ \\
\hline
\end{tabular}

As well as the PRESENT cipher with 128-bit key (see Fig. 8) shows time reductions from $14.8 \%$ to $25.3 \%$ for the version 2 and from $37.1 \%$ to $44 \%$ for the version 3, as shown in Table 4.

\section{CONCLUSIONS}

The time measurements performed on some common bit operations used in cryptographic algorithms implemented on Python 3 language, indicate that doing shifting operations spends at least $80 \%$ less time than the same operation performed by means of using the correspondent division in powers of 2. Likewise, another common bit operation is masking a specific group of bits inside a bigger group, for this the results indicates that masking the complete number of bits is at least a $15 \%$ faster than masking the specific bits and after shifting. It is important to highlight that these conclusion is applicable not only for the PRESENT but for any other cryptographic algorithm. The types of bit operations which shown time reductions were used to implement the three different versions of the PRESENT cipher developed in this paper.

Three different implementation approaches of the PRESENT cipher were developed and tested. The first one that uses individual bit reading and writing functions shown the largest execution time, the second one that does the $p$ Layer by means of using a shifting table reduced the time execution of the version 1 up to $31.9 \%$ for the 80 -bit cipher and up to $25.3 \%$ for the 128-bit one. Finally the last approach had the best time performance, this third version computes the different keys for all of the rounds previous to start the ciphering or deciphering processes; it shown a time reduction up to $45.3 \%$ for the 80-bit cipher and up to $44 \%$ for the 128-bit cipher. The obtained algorithm in Python language is able to be used on applications like IoT and/or smart sensor networks, along with embedded cryptographic hardware systems, optimizing the ciphering and deciphering times performed on the central computer.

Despite this implementation was designed to be run on a PC, the code can be run on any embedded system able to run a Python enterpreter. Nowadays the are some Python implementations which can run on microcontrollers such as: MicroPython, CircuitPython and Zerynth among others; all of those systems can be run subsets or even complete versions of Python 3. Another posibility is to use more complex embedded systems like the ones that are based on microprocessor instead of microcontroller (Raspberry $\mathrm{Pi}$, for instance), most of them use a linux kernel in which it is possible to install a complete version of Python 3. As a future work it is possible to compare the performance of the implemented PRESENT cipher running on some different kinds of embedded systems.

\section{NOMENCLATURE}

$x$

$S[x]$

$i$

$P(i)$
sBox Layer input

sBox Layer output

$\mathrm{p}$ Layer input bit position

p Layer output bit position 4-bit

4-bit

0 to 63

0 to 63 
$A$

$B$ input for the bit-wise operations

output for the bit-wise operations 128-bit

128-bit

\section{ACKNOWLEDGMENTS}

This work was completely supported by the Technology Faculty of Universidad Distrital Francisco José de Caldas. Authors thank SIE and ARMOS research groups for providing needed equipment to do the simulations and tests.

\section{REFERENCES}

1. Adomnicai, A., Berger, T. P., Clavier, C., Francq, J., \& ... (2019). Lilliput-AE: a new lightweight tweakable block cipher for authenticated encryption with associated data. Submitted to NIST .... https://csrc.nist.gov/csrc/media/projects/lightweightcryptography/documents/round-1/submissions/lilliput-ae.zip

2. Ahad, M. A., Tripathi, G., Zafar, S., \& Doja, F. (2020). IoT Data Management-Security Aspects of Information Linkage in IoT Systems. In Principles of Internet of Things (IoT) Ecosystem: Insight Paradigm (pp. 439-464). Springer.

3. Alcaraz, C. (2019). Security and privacy trends in the industrial internet of things. Springer.

4. Ali, A. (2017). Oppel-1: A new block cipher. 2017 14th International Bhurban Conference on .... https://ieeexplore.ieee.org/abstract/document/7868091/

5. Atiewi, S., Al-Rahayfeh, A., Almiani, M., Yussof, S., \& ... (2020). Scalable and Secure Big Data IoT System Based on Multifactor Authentication and Lightweight Cryptography. IEEE .... https://ieeexplore.ieee.org/abstract/document/9118946/

6. Azari, H. D., \& Dr. Prashant V Joshi. (2018). an Efficient Implementation of Present Cipher Model With 80 Bit and 128 Bit Key Over Fpga Based Hardware Architecture. 119(14), 1825-1832. https://acadpubl.eu/hub/2018-119-14/articles/3/61.pdf

7. Bansod, G., Patil, A., Sutar, S., \& ... (2016). ANU: an ultra lightweight cipher design for security in IoT. Security and .... https://onlinelibrary.wiley.com/doi/abs/10.1002/sec.1692

8. Bogdanov, A., Knudsen, L. R., Leander, G., Paar, C., \& Poschmann, A. (n.d.). PRESENT: An Ultra-Lightweight Block Cipher.

9. Eijs, H. (2020). Pycryptodome. In PYPi. https://pypi.org/project/pycryptodome/

10. Eisenbarth, T., Advisor, M., Sunar, B., Committee, T., Wyglinski, A., Committee, T., \& Mcneill, J. A. (2017). Threshold Implementations of the Present Cipher.

11. Ghafari, V. A., Hu, H., \& Chen, Y. (2016). Fruit-v2: ultra-lightweight stream cipher with shorter internal state. Int. Assoc. Cryptol. Res (IACR). https://pdfs.semanticscholar.org/f7c0/6c6c2d797568604276b60bb0d12d2fd0e743.pdf

12. Gnatyuk, S., Akhmetov, B., Kozlovskyi, V., Kinzeryavyy, V., Aleksander, M., \& Prysiazhnyi, D. (2019). New Secure Block Cipher for Critical Applications: Design, Implementation, Speed and Security Analysis. International Conference of Artificial Intelligence, Medical Engineering, Education, 93-104.

13. Gunduz, M. Z., \& Das, R. (2020). Cyber-security on smart grid: Threats and potential solutions. Computer Networks, 169, 107094.

14. Jithendra, K. B., \& Shahana, T. K. (2020). New Biclique Cryptanalysis on Full-Round PRESENT-80 Block Cipher. SN Computer Science, 1(2), 1-7.

15. Li, L, Liu, B., \& Wang, H. (2016). QTL: a new ultra-lightweight block cipher. Microprocessors and Microsystems. https://www.sciencedirect.com/science/article/pii/S0141933116300151

16. Li, Lang, Liu, B., Zhou, Y., \& Zou, Y. (2018). SFN: A new lightweight block cipher. Microprocessors and Microsystems, 60, $138-150$.

17. Muduvangattil, S. (2018). Review on PRESENT: An Ultra-Lightweight Block Cipher for Resource Constrained Devices. Recent Trends in Analog Design and Digital .... http://hbrppublication.com/OJS/index.php/RTADDD/article/view/486

18. ÖzÇakmak, B., Özbillen, A., \& ... (2019). Neural and Quantum Cryptography in Big Data: A Review. ...on Big Data (Big Data). https://ieeexplore.ieee.org/abstract/document/9006238/ 
19. Rahouma, K. H., AbdelGhany, F. M., Mahdy, L. N., \& Hassan, Y. B. E. (2020). Design and Implementation of a New DNA Based Stream Cipher Algorithm using Python. Egyptian Computer Science Journal, 44(1).

20. Setetemela, K. O., Keta, K., Nkhabu, M., \& Winberg, S. (2019). Python-based FPGA implementation of AES using Migen for Internet of Things Security. 2019 IEEE 10th International Conference on Mechanical and Intelligent Manufacturing Technologies, ICMIMT 2019, August, 194-198. https://doi.org/10.1109/ICMIMT.2019.8712074

21. Sutanto, L. (2019). The privacy preserving framework with virtual ring and identity-based cryptography for Smart Grid. academicworks.cuny.edu. https://academicworks.cuny.edu/ny_pubs/548/

22. Zhao, H., Yang, F., Cui, Y., Yang, R., Pan, D., \& ... (2020). Design of a New Lightweight Stream Cipher VHFO Algorithm. 2020 3rd International .... https://ieeexplore.ieee.org/abstract/document/9131221/ 